\title{
EGADS approaching GADZOOKS!
}

\author{
Lluís Martí Magro1,a \\ ${ }^{1}$ University of Tokyo, Japan
}

\begin{abstract}
The detection of the diffuse supernova neutrino background (DSNB) for the very first time and its difficulty due to the large irreducible backgrounds originally motivated the idea of adding gadolinium (Gd) into the ultra-pure water of Super-Kamiokande (SK). Since the main mode to detect the DSNB is through inverse beta decay, these backgrounds could be greatly reduced if neutrons could be detected with high efficiency. Today, this is not currently possible. Adding Gd was proposed by GADZOOKS! about 10 years ago and since then much research has been done towards achieving this goal. Given the many advantages that adding Gd means, the EGADS project was set in 2009 to Evaluate the Gadolinium's Action on Detector Systems. Soon we will be able to demonstrate that this technique is possible, feasible at reasonable costs and safe making possible the advantages of reducing backgrounds in several analyses at SK or in future experiments like Hyper-Kamiokande.
\end{abstract}

\section{Core-collapse Supernova and beyond}

Big stars' life undergo different stages of nuclei fusion: first hydrogen, then helium, and so on until they start burning silicon. At that stage, a core of nickel and iron that cannot be further burned is formed. Not being able to balance the huge gravitational pressure by further fusing nuclei, the star collapses. Core-collapse supernovae $(\mathrm{ccSN})$ are among the most energetic phenomena in the universe releasing on the order of $10^{46} \mathrm{~J}$. The explosion in the Milky Way of one of these stars is a very bright event, it can be brighter than any other star (apart from our Sun) and even be visible during the day. But the most striking thing is that about $99 \%$ of its energy is released as neutrinos (about $10^{58}$ ) and not as light. In 1987, a ccSN in the Large Megallanic cloud occurred (about 50 Kpc). Kamiokande II, IMB and Baksan detectors recorded 11, 8 and 5 anti-neutrinos respectively, and to this date they are the only neutrinos known to be coming from a ccSN. These few events were enough to confirm the general understanding behind the theoretical models about ccSN explosions.

If a ccSN would occur now in our galaxy many more events could be recorded at SK (10000 events at $10 \mathrm{kpc}$, the distance to the galaxy centre). Precise measurement of the neutrino energy spectrum and time sequence could be done to further constrain our current models. Because neutrinos interact with matter only weakly they can be detected earlier than the light. They can therefore be used to predict the SN optical burst. Adding Gd would give us significant extra information:

- It will allow to identify the dominant (about 89\%) inverse beta decay events (IBD): $\bar{v}_{e}+p \rightarrow e^{+}+n$. Being able to identify the IBD events will allow us to study the other reactions such as ${ }^{16} \mathrm{O} \mathrm{NC}$ or $\mathrm{O}\left(v_{e}, \mathrm{e}^{-}\right) \mathrm{F}$ events.

\footnotetext{
ae-mail: martillu@ suketto.icrr.u-tokyo.ac.jp
} 
- Neutrino elastic scatterings $v+e^{-} \rightarrow v+e^{-}$allow us to determine the direction in the sky where the neutrinos came from and hence the ability to point our optical telescopes towards the SN before it is visible. Therefore, being able to identify the much larger IBD background events to this reaction will also improve the pointing accuracy.

- Neutrino-cooled stars produce neutrinos and anti-neutrinos of low energies (about $2 \mathrm{MeV}$ ). For very massive stars of $8 \mathrm{M}_{\odot}$ and during Si burning, the production of these neutrinos could be enough to be detected depending on its distance: while the neutrinos are not possible to detect due to its low energy, anti-neutrinos could be detected through IBD. Although the positron would still be below the Cherenkov threshold, the neutron could still be detected in Gd loaded water Cherenkov detectors. The rise in trigger rates from neutron captures would be a signal of a forthcoming ccSN. Because of this, we would keep the detector live-time as high as possible to make sure we are ready for the ccSN neutrino burst.

The neutrinos from core-collapse supernovae we can detect with our current detectors are ccSNe in our galaxy and only two or three ccSN per century are expected. For ccSNe far from our galaxy the neutrino flux is too small. However, there have been many ccSN in the history of the universe and the copious and ubiquitous neutrinos produced in them constitute the diffuse supernova neutrino background (DSNB). Detecting DSNB for the first time would be an important milestone in the neutrino astronomy, since only two sources of astronomical neutrinos are known so far: the Sun and SN1987a. Moreover, the discovery of DSNB would rule out neutrino decay scenarios or at least severely constrain them. With enough statistics, we could not only learn more about the ccSN mechanism but also about the history of our universe itself. Despite the many efforts at SK this analysis is still background dominated although we could set the best world limits on the DSNB flux [1].

\section{EGADS towards GADZOOKS!}

The motivation of GADZOOKS! [2] to dope the SK ultra-pure water with a Gd salt has its roots in the DSNB search. It was proposed by J. F. Beacom and M. R. Vagins in 2003 and soon thereafter investigations for its application at SK started. Since the current SK water filtration system would remove all Gd in water, the first challenge was to develop a water filtration system that could be used with Gd loaded water. Vagins developed a small scale filtration system where the Gd retain rate was over $99.9 \%$ per pass.

In 2009 a R\&D project was funded to give answer to all the remaining questions in order to load SK with Gd. The project EGADS (Evaluate the Gadolinium's Action on Detector Systems) is located in a new hall near the SK detector in the Kamioka mine. The project has five clear goals: it has to demonstrate that the filtration system can achieve and maintain a good water quality while keeping Gd, it has to show that Gd sulfate has no adverse effects on the detector components at SK (we use the same materials that were used at SK only), it has to show we can add/remove Gd in a efficient and economical way and finally, how to reduce the now visible neutron background (from spallation, U/Th fission chains and ambient neutrons, etc) and show it does not affect the SK analyses. It initially featured a 200-ton tank, a pre-treatment system, a filtration system based on Vagins previous experience, a device to measure water transparency and an atomic absorption spectrometer (AAS).

The 200-ton tank is made of the same stainless steel as the SK tank. Initially, the 200-ton tank was not instrumented. However, it had the needed stainless steel structure for the later installation of PMTs. The compensation coil to reduce the Earth's magnetic field inside the tank was also prepared. The PMTs were installed in the summer of 2013. 
The pre-treatment system mainly consists of a 15-ton plastic tank with a stirrer to dissolve Gd, micro-filters to remove dust and other contaminants in the Gd sulfate and a special resin (AJ4400) to remove uranium while keeping $\mathrm{Gd}$.

The filtration system is a larger scale version of the smaller filtration system developed by Vagins at the University of California, Irvine (UCI). Later, other elements were added, e.g. vessels with AJ4400 were also added since experimentally it has been seen that it improves the water quality. Another very important related system is the membrane flushing system: the membranes need to be flushed far beyond the manufacturer's recommendations in order to get the proper membrane conditioning and do not degrade the water transparency.

A device to monitor the water transparency was developed at UCI. The UDEAL device (Underground Device Evaluating Attenuation Length). It consists of a beam injector with seven wavelengths (one used at each time), whose light is split into two: part of the beam is used to monitor the beam intensity with an integrating sphere and part of it is steered through a pipe partially filled with water and finally monitored (integrating sphere). The pipe is filled with water at several heights and thus, the water transparency can be calculated for the seven wavelengths.

\section{Past, Present and Future}

\subsection{Past}

After the new cavern and the new equipment was prepared and installed, the first goal was to test the performance of the new water filtration system with pure water. In the first half of 2011 this test was successfully performed: the water filtration system soon achieved SK water transparency levels [3].

The next step was to test the water filtration system performance with Gd loaded water. These tests were done the second half of 2011 until end of 2012 [4]. For this purpose, the 15-ton plastic tank of the pre-treatment system was used. The were two main advantages: first, the test of interaction of Gd and stainless steel could be postponed for a later stage and test the performance of the filtration system only and second, the smaller volume of this tank meant faster water recirculation which allowed us to perform several tests and observe its effects much faster. With this test we learnt key effects of Gd loaded water flow through our system and how to set our filtration system in order to achieve high cleaning performance with a high Gd retention efficiency. We also learnt how to condition our membranes whenever we have to substitute them with new one. The AJ4400 resin was initially installed in the pre-treatment system to remove uranium. However, another important lesson was to realise that it is also able to remove other impurities and improve the water transparency too. Figure 1 shows the Cherenkov light left after $15 \mathrm{~m}$ when loading water with $0.2 \% \mathrm{Gd}$ in mass in the 15 -ton tank. This is a convolution of the Cherenkov spectrum and the attenuation length in Gd loaded water and the quantum efficiency of the PMTs for each frequency.

The Gd concentration was also measured to monitor the possible Gd loss in our water purification system with an atomic absorption spectrometer (AAS). No Gd loss could be detected and we could estimate that the efficiency retaining Gd was above $99.9 \%$.

In the fist half of 2013, we performed the same test with the bare stainless steel 200-ton tank. Injecting $\mathrm{Gd}$ into the tank is done stepwise in batches. At the beginning with batches of $30 \mathrm{Kg}$ and later with $60 \mathrm{Kg}$ ones. During the full process of injecting Gd, not only the water transparency was measured as we did before but also the Gd concentration in water was constantly monitored with an AAS.

In figure 2 the concentration of gadolinium sulfate $\left(\mathrm{Gd}\left(\mathrm{SO}_{4}\right)_{3}+\mathrm{n} \cdot \mathrm{H}_{2} \mathrm{O}\right)$ in the 200-ton tank as a function of time is shown. As for the water transparency measurements, samples are taken at three different positions: top, centre and bottom. The injections can be seen as jumps in the concentration. 


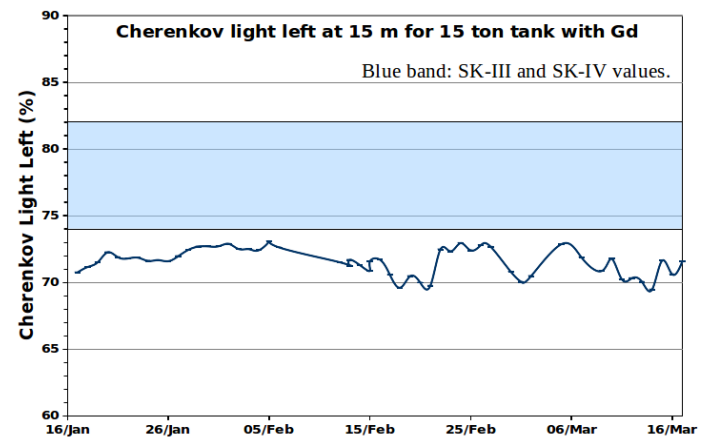

Figure 1. Cherenkov light left at $15 \mathrm{~m}$ as a function of time. The blue band shows the typical SK-III and SK-IV water transparency values. Note the zero suppressed y-axis.

If the tank is not further loaded the concentration stays constant and homogeneous. Since the loaded water is injected from the bottom, note the delayed increase of concentration in the centre and specially top positions.
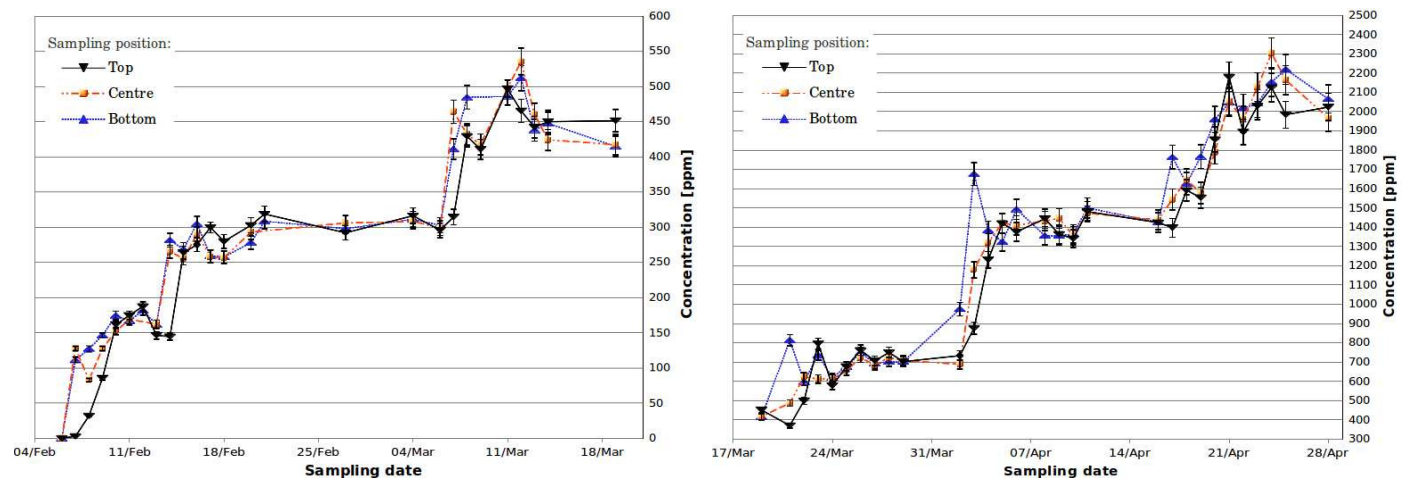

Figure 2. Gadolinium sulfate concentration $\left(\mathrm{Gd}\left(\mathrm{SO}_{4}\right)_{3}+\mathrm{n} \cdot \mathrm{H}_{2} \mathrm{O}\right)$ as a function of time in the three sampled positions: top, centre and bottom. The final concentration of gadolinium sulfate is about $0.2 \%$ in mass (equivalent to $0.1 \%$ of $\mathrm{Gd}$ ). The left figure shows single loads of $30 \mathrm{Kg}$ while the right figure shows the double ones of 60 $\mathrm{Kg}$ (note here the suppressed zero on the $\mathrm{y}$-axis.

As in previous tests, the water transparency was also monitored. see figure 3. Before injecting $\mathrm{Gd}$ the water transparency was very high and in the upper region of typical SK-III and SK-IV values (blue band in the figure). The Gd injections are marked with vertical hatched lines and their width show their duration (the total concentration is also shown with a final value of $0.2 \%$ in mass). After each injection the water transparency decreases. However, in the last ones it seems to decrease less than in the first ones although the load was double. After the last load the CLL remains constant and about $69 \%$, a value very close to the typical SK water transparency values and very similar to the 15 -ton tank test final value.

After this test, the about $400 \mathrm{Kg}$ of diluted Gd sulfate had to be disposed (this represents about 200 $\mathrm{Kg}$ of $\mathrm{Gd}$ ). Before releasing the Gd loaded water into the environment the tank was drained through 


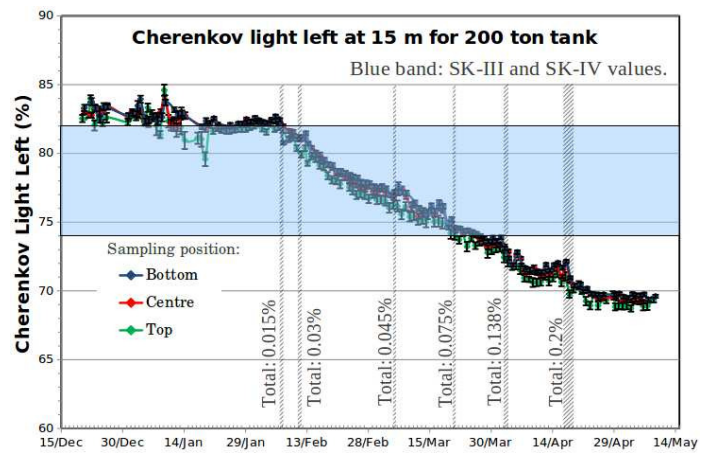

Figure 3. Cherenkov light left at $15 \mathrm{~m}$ as a function of time for the three sampling positions: top, centre and bottom. Vertical hatched lines indicate the Gd injections and their widths their duration. The blue band shows the typical SK-III and SK-IV water transparency values. Note the zero suppressed y-axis.

a special resin that captures the Gd. After being processed the water contains less than $0.5 \mathrm{ppb}$ of Gd. After draining the 200-ton tank and processing the Gd loaded water the resin was disposed. However, there are methods to recover the resin and reuse it again and thus, making this process economically possible when going to SK scales.

After draining the 200-ton tank we proceeded to install the 240 photodetectors in summer 2013. Most of them, 227 in total, were the 50-cm Hamamatsu PMTs (R3600), the same PMT type that is currently installed in SK. Due to insufficient spares from SK construction, only some of them have been installed in $10 \mathrm{~mm}$ fibreglass (FRP) rear and $13 \mathrm{~mm}$ acrylic front covers, while others were installed in FRPs only or un-housed. Besides the SK-like PMTS, 8 20-cm hybrid-photodetectors (HPD) and 5 50-cm high quantum efficiency (QE) PMTs that are being developed for Hyper-Kamiokande were installed [5]. The photocoverage is similar to SK (about $40 \%$ ). Apart of the size, one important difference of the HPD as compared to a SK-like PMT is that instead of dynodes has an avalanche photodiode. Another very important feature is the higher QE of the HPDs and the higher single photon sensitivity and higher timing resolution.

As in SK, opaque black polyethylene telephthalate sheets were installed to cover the gaps between PMTs to suppress low-energy radioactive events occurring behind the PMTs to leak in the detector.

A DAQ system with the same front-end electronics as SK before 2008 has been also prepared. Along with the DAQ, calibration procedures were also prepared, e.g.: 1 photo-electron peak measurement, relative timing and relative gain calibration as well as auto-Xe lamp + scintillation ball to monitor gain variations and periodic $\mathrm{Am} / \mathrm{Be}$ source deployments to monitor the neutron capture on Gd efficiency.

In parallel to the above tests, measurements of radioactive contamination in the Gd sulfate itself has been measured with germanium detectors at the Canfranc Underground Laboratory, Spain, by members of our group at the Universidad Autonoma de Madrid. Since the radioactive contamination will be diluted together with the Gd sulfate in the whole detector it is important to keep this contamination as low as possible. For this purpose, several batches of Gd sulfate of several manufacturers have been tested. Typical values of the selected ones are shown in table 1. Taking into account the dilution factor in water and that the AJ4400 resin is able to remove $\mathrm{U}$, the background for the DSNB becomes $<3 \cdot 10^{-2}$ events/year at SK as compared to the expected value of about 5 events/year. For the solar neutrino analysis, the background is estimated to be $5 \cdot 10^{3}$ events/day/kton mainly from 
Table 1. Typical values of radioactive contamination in our selected Gd compounds.

\begin{tabular}{ccc}
\hline chain & sub-chain & $\mathrm{mBq} / \mathrm{Kg}$ \\
\hline${ }^{238} \mathrm{U}$ & ${ }^{238} \mathrm{U}$ (upper) & 50 \\
& ${ }^{226} \mathrm{Ra}$ (lower) & 5 \\
\hline${ }^{232} \mathrm{Th}$ & ${ }^{228} \mathrm{Ra}$ (upper) & 10 \\
& ${ }^{228} \mathrm{Th}$ (lower) & 100 \\
\hline${ }^{235} \mathrm{U}$ & ${ }^{235} \mathrm{U}$ (upper) & $<30$ \\
& ${ }^{227} \mathrm{Ac}$ (lower) & 300 \\
\hline
\end{tabular}

${ }^{208} \mathrm{Tl} \beta$-decays as compared to the solar $v_{e}$ flux of about 10 events/day/kton in the $3.5 \mathrm{MeV}$ to $5 \mathrm{MeV}$ kinematic energy range.

\subsection{Present}

After installing the PMT installation the detector was filled with pure water. Unlike previous tests we experienced difficulties in keeping the water quality at SK levels. While at first we considered this to be expected due to the recent work inside the tank during PMT installation, it was the first symptom of something not going properly. Despite this unrecognised warning, we went on with the first Gd injection which triggered a clear deterioration of the water quality. When draining the tank again, the source of contamination was readily found: a wire used to support the black sheet was not made of the required stainless steel quality.

The wire was removed and the detector has been refurbished in summer 2014. The detector was cleaned to remove the rust left by the wire.

As of beginning of November 2014 we are running again with pure water and finishing the detector calibration. The next immediate next step will be to load the detector with Gd again. With this exhaustive refurbishment we expect to successfully complete this last step in the R\&D phase of the EGADS project.

\subsection{Future}

After finishing the R\&D phase a new life for the EGADS detector will start as a galactic SN detector: the first Gd based SN detector in the world. Before this becoming a reality we need to replace the current Analog-Timing-Modules with QTC (charge-timing-converter) Based Electronics with Ethernet (QBEE). They provide higher sensitivity for single photoelectrons, a wider charge dynamic range (about 4-5 times that of the the ATM modules) and good timing resolution for the 50-cm PMTs.

With these new electronics EGADS will become the world's first Gd-based Cherenkov detector with full galactic supernova neutrino sensitivity. In case of SN, the EGADS detector will immediately and without any doubts allow us to detect it: the delayed neutron captures on Gd following the prompt positrons will be the proof without a doubt of an ongoing SN.

\section{References}

[1] Bays, K. et al. Phys.Rev.D 85052007 (2012).

[2] Beacom, J.F. and Vagins, M.R. Phys.Rev.Lett. 229-232 514 (2004).

[3] Marti Magro, Ll. [Super-Kamiokande Collaboration] 32nd ICRC Proceedings 4 (2011) 234

[4] Marti Magro, Ll. [Super-Kamiokande Collaboration] 33rd ICRC Proceedings 4 (2013) 234

[5] Hirota, S. et al. [Hyper-Kamiokande Working Group] Nucl.Instrum.Meth.A732, 303-305 (2013) 